\title{
Observation Unit Experience for Pediatric Poison Exposures
}

\author{
Diane P. Calello, MD ${ }^{a, b}$, Elizabeth R. Alpern, MD, MSCEc, Maureen McDaniel-Yakscoe, CRNPd, \\ Brianna L. Garrett, MDe, Kathy N. Shaw, MD, MSCEc, Kevin C. Osterhoudt, MD, MSCEf,g
}

\author{
aSection of Medical Toxicology, Division of Emergency Medicine, Department of Pediatrics, Robert Wood Johnson Medical \\ School-University of Medicine and Dentistry of New Jersey, New Brunswick, NJ \\ bNew Jersey Poison Information and Education Systems (NJPIES), Newark, NJ \\ cDepartment of Pediatrics, Division of Emergency Medicine, The Children's Hospital of Philadelphia, University of Pennsylvania \\ School of Medicine, Philadelphia, PA \\ dDepartment of Nursing, The Children's Hospital of Philadelphia, University of Pennsylvania School of Medicine, \\ Philadelphia, PA \\ eDepartment of Emergency Medicine, University of Pittsburgh School of Medicine, Pittsburgh, PA \\ fSection of Clinical Toxicology, Division of Emergency Medicine, Department of Pediatrics, The Children's Hospital of \\ Philadelphia, University of Pennsylvania School of Medicine \\ gThe Poison Control Center, Philadelphia, PA
}

\begin{abstract}
Background: Short-Stay Emergency Department Observation Units (OU) are an alternative to hospitalization, but data on OU care of pediatric poisoning exposures is limited. We report the experience of a pediatric OU with this population.

Methods: We retrospectively reviewed the charts of children with poison exposure admitted to a pediatric OU during a 30-month period. Data was collected pertaining to demographics, type of exposure, clinical presentation, and rate of hospitalization, and was compared to nonpoisoned OU patients.

Results: Of the 91 pediatric patients with poison exposure, 86 complete charts were available for review (94.5\%). Of these patients, $49.5 \%$ were female, and $82.4 \%$ were $<6$ years of age (range 1.5 months to 16.6 years). There were a total of 98 toxicants implicated, the most common of which were psychoactive drugs (25\%) and cardiovascular agents (19\%). At OU admission, 33 of 88 patients (38\%) had altered mental status or abnormal vital signs. Only 2 of the 53 remaining patients developed abnormal vital signs within the OU. Two patients were hospitalized unexpectedly with respiratory distress due to hydrocarbon and charcoal aspiration pneumonitis, respectively; the unexpected hospitalization rate was $2.2 \%$. Three more planned hospitalizations for endoscopy or psychiatric evaluation led to a total hospitalization rate of 5.4\%. This hospitalization rate is significantly lower $(\mathrm{RR}=0.26,95 \% \mathrm{CI}=0.11-0.62)$ than the hospitalization rate from the OU for nonpoisoned patients (20.3\%) during that time. Mean OU length of stay for nonadmitted poisoned patients was 14.35 hours. There were no adverse events noted as a result of OU placement.
\end{abstract}

Conclusion: Select poisoned pediatric patients appear suitable for OU management and had less frequent unexpected hospitalization from the OU than other diagnoses.

Keywords: observation, pediatrics, poisoning

Notes: The contents of this study were presented in abstract form at the May 2005 meeting of the American Academy of Pediatrics/Pediatric Academic Societies in Washington, DC.

There was no outside funding of any kind used for this study.

Corresponding Author: Diane P. Calello, MD, Robert Wood Johnson Medical School-UMDNJ, Department of Pediatrics, One Robert Wood Johnson Place, PO Box 19, New Brunswick, NJ 08903-0019.E-mail: diane.calello@umdnj.edu 


\section{INTRODUCTION}

Observation units (OUs), also known as Short-Stay Wards or Outpatient Extended Treatment Sites, provide an alternative evaluation and management site for patients with select diagnoses for a defined time period of usually less than 24 hours. OUs serve a variety of functions, such as extending the evaluation period for patients potentially needing inpatient admission; making short-term interventions and observations in common illnesses; and attending to patients undergoing elective procedures or awaiting social, psychiatric or other consultation services, or for whom an inpatient bed is not yet available $[1,2]$. At any time during the OU stay, if a patient's clinical picture worsens or it becomes obvious that the patient will require an admission lasting longer than 23 hours, full hospital admission may be arranged. Avoiding hospitalization by utilization of an OU may decrease total associated medical costs while increasing emergency department (ED) revenue. OUs have the ability to improve the quality of care given to patients for the reasons stated above and may reduce inpatient admissions, liability, and healthcare costs [3-5]. A national survey of 522 hospitals in 1999 revealed that $19 \%$ had an OU, $64 \%$ of which admitted pediatric patients, and another $12 \%$ planned to open an OU [6]. Although pediatric OUs have existed since the 1970s and are increasing in prevalence, a limited number of studies have been published describing the types of patients admitted to pediatric OUs and their disposition outcomes [7-10].

Much of the existing literature has sought to identify the ideal patients for OU care. In general, these are patients who would not otherwise be discharged from the ED, but who are expected to require less than 24 hours of hospital care. Poisoned patients can fall into this model using the OU as a "safety net" to evaluate evolving toxic syndromes. Because children comprise over half of annual poison exposures reported, there is a need to determine the efficacy and safety of using OUs for poisoned pediatric patients. The objective of the current study was to examine the use of a tertiary care Pediatric Emergency Department (PED) observation unit in the care of select poison exposures.

\section{METHODS}

An observational cohort of consecutive patients with poison exposure were evaluated in the PED and admitted to the OU during a 30-month period from January 1999 through June 2001. Each subject had a data questionnaire prospectively completed at admission to and discharge from the 23 -hour OU by physicians, nurse practitioners, and nurses caring for the patient. Data elements prospectively collected included date of birth, gender, race/ethnicity, insurance type, triage acuity, primary ED diagnosis, disposition, treatment(s) received in 23-hr OU, as well as date and time of triage, arrival in ED, 23-hr OU admission, and 23-hr OU discharge [11]. In addition, we retrospectively reviewed the charts of these prospectively identified patients for additional clinical information specifically pertaining to the poisoning presentation, treatment, and diagnosis.
The Institutional Review Board of The Children's Hospital of Philadelphia approved this study. The study setting was the 23-hr $\mathrm{OU}$ at a freestanding urban tertiary care pediatric hospital.

Patients were selected for OU admission by the ED attending physician. All patients were initially treated and evaluated in the ED; there were no direct admissions to the OU from outpatient clinics or non-ED hospital settings. Explicit criteria for suitable patients were not used at the time of the study; however, a general set of guidelines was in operation, as suggested by the American College of Emergency Physicians and other authors [10,12]. Although not specifically tailored to poisoned patients, these criteria include 1) clearly identified patient care goals, 2) limited severity of illness or need for intensive care, 3) anticipation of discharge within 24 hours, and 4) clinical condition appropriate for observation. OU care was provided by Pediatric Emergency Medicine fellowship-trained attending physicians or Urgent Care attending pediatricians in the Division of Emergency Medicine, in conjunction with pediatric nurse practitioners; the unit had in-house attending physician coverage 24 hours a day. All clinicians had access to poisoning information provided by a certified regional poison control center associated with the hospital.

Computerized and written medical records were obtained and abstracted by one investigator (DC). Data was collected pertaining to demographics, type of exposure, mental status and vital signs at presentation in the PED and OU, duration of stay in OU, OU course, disposition (discharge home or inpatient hospital admission), and revisit to our ED within 48 hours. Poison exposures were classified by medication or toxicant class, as defined by the American Association of Poison Control Centers (AAPCC) [13]. In the event that a patient had more than 1 toxicant exposure, all exposures were accounted for in our description and statistics. A "worsened" clinical course was defined as deterioration in vital signs or mental status from previously documented normal values at time of OU admission, or initially abnormal vital signs and/or mental status that failed to improve while in the OU. The overall OU hospitalization rate for poisoned patients included both those with a worsened clinical course and those admitted for continued medical care beyond 24 hours. The unexpected hospitalization rate included only those patients whose clinical status worsened; patients admitted for "planned" evaluation (endoscopy, psychiatric consultation) not available in the ED were not included.

Descriptive statistics were determined to define the study population; means and standard deviations were used for continuous variables, and discrete variables were described as counts and percentages. Incidence rates of hospitalizations were calculated with a 95\% confidence interval. Comparisons were made to nonpoisoned OU patients with Fisher's Exact test. All statistical analyses were performed on Stata Version 6 (College Station, TX).

\section{RESULTS}

A total of 4453 children, comprising $2.9 \%$ of the total ED volume, were admitted to the OU during the 30-month study period. 


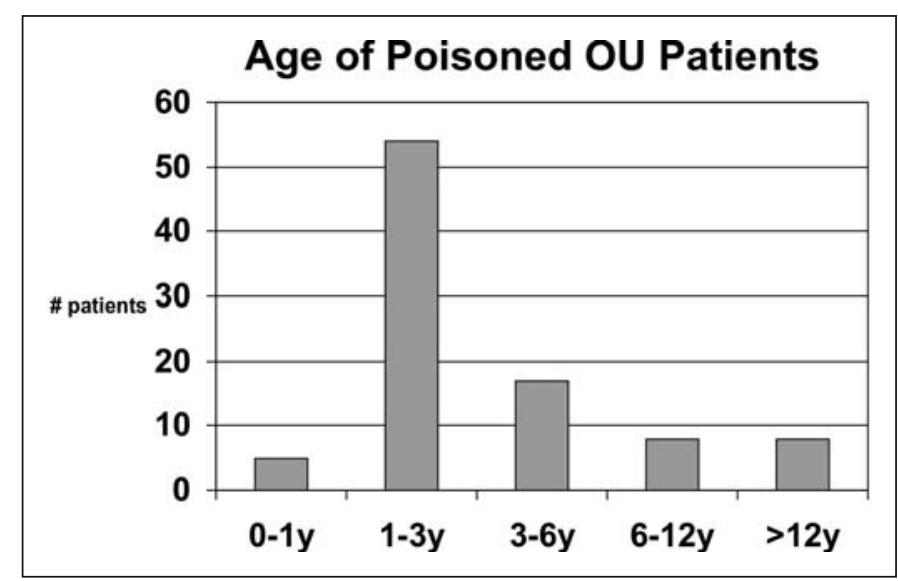

Figure 1: Age distribution of poisoned patients admitted to the observation unit.

Table 1: Exposure by Substance Class

\begin{tabular}{|c|c|c|}
\hline Substance Class & $\begin{array}{c}\mathbf{N}(\% \text { of } \\
\text { exposures) }\end{array}$ & Examples \\
\hline $\begin{array}{l}\text { Psychoactive } \\
\text { medications }\end{array}$ & $25 / 98(25.5 \%)$ & $\begin{array}{l}\text { Antidepressants, } \\
\text { antipsychotics, sedative/ } \\
\text { hypnotics }\end{array}$ \\
\hline $\begin{array}{l}\text { Cardiovascular } \\
\text { medications }\end{array}$ & $19(19.4 \%)$ & $\begin{array}{l}\text { Beta blockers, calcium- } \\
\text { channel blockers }\end{array}$ \\
\hline $\begin{array}{l}\text { Cough/cold } \\
\text { preparations }\end{array}$ & $10(10.2 \%)$ & $\begin{array}{l}\text { Antihistamines, } \\
\text { decongestants }\end{array}$ \\
\hline Cleaning substances & $8(8.2 \%)$ & $\begin{array}{l}\text { Ammonia/bleach, caustic } \\
\text { cleansers }\end{array}$ \\
\hline Anticonvulsants & $3(3.1 \%)$ & $\begin{array}{l}\text { Topiramate, phenobarbital, } \\
\text { carbamazepine }\end{array}$ \\
\hline $\begin{array}{l}\text { Gastrointestinal } \\
\text { preparations }\end{array}$ & $5(5.1 \%)$ & $\mathrm{H} 2$ blockers, prokinetics \\
\hline Hydrocarbons & $4(4.1 \%)$ & Lamp oil, pine oil \\
\hline Cosmetics & $4(4.1 \%)$ & "No-lye" hair relaxers \\
\hline Other & $6(6.1 \%)$ & $\begin{array}{l}\text { Aspirin }(1 \%), \text { vitamins (1\%), } \\
\text { hypoglycemics }(1 \%) \text {, local } \\
\text { anesthetics }(1 \%), \\
\text { bromocriptine }(1 \%) \text {, felt- } \\
\text { tipped pens }(1 \%)\end{array}$ \\
\hline
\end{tabular}

Ninety-one patients ( $2 \%$ of total) were poison exposures, and of these, complete data was available on 86 patients (94.5\%). Data was available on all 91 patients pertaining to demographics, length of stay, and disposition: $49.5 \%$ were female, $82 \%$ of patients were $<6$ years of age (range 1.5 months to 16.6 years, median 2.3 years) (Figure 1). Length of stay and disposition results are reported below.

Table 1 presents a list of poison exposures by substance class as described by the AAPCC [13]. There were 98 exposures in 86 patients (some patients reported more than 1 exposure), the most common of which were psychoactive drugs $(25 / 99,25.3 \%)$ and cardiovascular agents (19/99, 19.2\%). In 6/98 (6.1\%) instances, the type of exposure could not be determined and these patients were placed in an "unknown" category.

At the time of OU admission, 33/88 (38\%) of patients had altered mental status or abnormal vital signs for age. A small proportion, 5/86 (6\%), had both altered mental status and abnormal vital signs. All 33 patients with initial altered mental status or abnormal vital signs had normalization of both of these parameters during the OU stay. One of these patients was hospitalized for a planned endoscopy following a caustic ingestion, while the remaining 32 were discharged from the OU.

Only 2 patients, or $4 \%$, of the 53 patients with normal mental status and normal vital signs ( $2 \%$ of the total 86 exposures for whom full data was available), developed abnormal vital signs within the OU. These patients' progression to respiratory distress was due to hydrocarbon and charcoal aspiration pneumonitis, respectively, and led to unexpected hospitalizations from the OU. Three more planned hospitalizations for endoscopy or psychiatric treatment (one of which is described above) led to a total hospitalization rate of 5.5\% (5/91) and an unexpected hospitalization rate of $2.2 \%(2 / 91)$. This admission rate is significantly lower $(\mathrm{RR}=0.11,95 \% \mathrm{CI}=0.03-0.42)$ than the nonpoisoned OU admission rate $(20.3 \%)$ during that time. Mean length of stay for nonadmitted poison patients was 14.35 hours ( \pm 6.6 hours), which was similar to the overall OU length of stay at 15.0 hours $( \pm 5.9$ hours). There were no adverse events noted as a result of admission to the OU.

\section{DISCUSSION}

The pharmacokinetic parameters of absorption, distribution, and elimination for most toxicants lead to somewhat predictable toxicodynamics. Therefore, poisoned patients, many of whom require care only for a short time, can be ideal candidates for observation. The OU can also provide an area where the patient awaits consultants who will address emotional and psychiatric needs, as well as home and workplace safety [14]. In patients with exposures to unknown or delayed release medications, such as oral hypoglycemics and sustained-release cardiac preparations, observation services can help discover and manage any delayed complications [14]. Patients with poison exposures have been reported to be among the most common diagnoses admitted to OUs and the most common to successfully attain discharge within the 23-hour observation period [10]. Children comprise greater than half of all poison exposures reported annually; although many of these exposures are inconsequential and not treated in healthcare facilities, those that are referred to healthcare facilities in general have better outcomes than their adult counterparts, making them intriguing candidates for short-stay care [13]. There is some emerging data regarding these children with poison exposures, who accounted for about $4 \%$ of all OU admissions in prior studies $[7,9,10]$. Scribano et al. and Zebrack et al. reported low subsequent hospitalization rates from their respective OUs for poisoned patients $[9,10]$. Two studies in adults 
focused on intoxications in the OU and separately concluded that the $\mathrm{OU}$ is a valuable resource for safely managing poisoned patients $[15,16]$.

Interestingly, in our study children younger than 6 years of age represented the majority of exposures. Although this is in keeping with national poison center data reported annually, the typical bimodal distribution (with a second peak in adolescence) was not seen in this study. This may be due to the more serious nature of adolescent ingestions, which are more likely to be suicidal in nature and therefore more medically consequential and more readily deemed unsuitable for OU care [10].

Considering that it is often unknown exactly what type(s) or amount of a particular substance or medication a child has ingested, or if an exposure has truly occurred, a period of observation becomes particularly relevant in pediatric emergency medicine. In our population, there were 6 unknown exposures, all of whom were discharged from the OU, and 4/6 that initially had abnormal mental status and improved to baseline. This group appears well suited to the observation unit based on this series.

In our study, we found that all 33 (38\%) of those with initially abnormal vital signs or mental status were successfully discharged from the OU in less than 24 hours. Two (4\%) of the initially stable poisoned patients developed worsening of symptoms requiring inpatient admission, and 3 others were admitted for a planned endoscopy or psychiatric treatment, resulting in a total admission rate of $5.4 \%$ from the OU. The OU stay for patients waiting for "planned admission" for nonemergent operating room or endoscopy suite utilization and then potential subsequent same-day discharge of a patient with minimal needs during the 23-hr OU stay is notable and may provide useful information for many units. We established that this overall admission rate for poisoned patients (5.4\%) was significantly less than the admission rate from the OU of nonpoisoned patients.

Our results are comparable to prior studies that reported hospital admission rates for the poisoned patient from the OU of $4 \%$ and $5 \%$, respectively $[9,10]$. Zebrack et al. describes a total hospitalization rate from the OU of $15 \%$ [10]. Scribano et al. compared pediatric poisonings to the remaining 4 most common diagnoses in their OU, and demonstrated that poisonings had the lowest subsequent need for admission (4\%) compared to seizures (19\%) as well as the other most common diagnoses (asthma, croup, and enteritis/dehydration) that were admitted at an average rate of $10 \%$ [9]. Two abstracts examining the use of adult OUs report an inpatient hospitalization rate of $6 \%$ and $8 \%$, respectively, of poisoned patients from the OU $[15,16]$.

The results of our study of poisoned patients in a pediatric OU are similar to the experiences of the other studies described and support the utilization of an OU for select pediatric poisoned patients. We agree with previous authors who have recommended a multidisciplinary approach with routine toxicology consultation in the management of these patients [14].

\section{Limitations}

One limitation of this study is its retrospective design and reliance on information available in the patient's chart. Suitability of patients for the OU was determined by attending physician judgment, which may not generalize to all settings and so may have introduced selection bias. We do not have prospectively documented information concerning poisoned patients admitted directly to the inpatient service or discharged directly from the ED for comparison to those treated within the OU. Therefore, a limitation of this study includes lack of information concerning the clinical thought process of practitioners as they admitted patients to the 23-hour OU. This information may have distinguished additional factors that would influence the unexpected hospitalization rate. However, research concerning treatment decision factors may be best accomplished in a qualitative manner and should be addressed in the future. In addition, we do not have information on the clinical course of patients after discharge from the OU or on admission to the inpatient care unit. It is conceivable that some patients that we considered "successful" discharges from the OU returned to the ED or presented to another ED within a brief period of time. Also, some patients with unanticipated admissions from the OU may actually have been discharged from the inpatient units in a very timely manner. Last, we did not evaluate other outcomes such as patient satisfaction or perform a formal economic analysis of resource utilization.

\section{CONCLUSION}

Select pediatric poisoned patients appear suitable for OU management and had unexpected hospitalizations from the OU less frequently than patients with other diagnoses.

The authors have no potential financial conflicts of interest to report.

\section{REFERENCES}

1. Mace SE. Pediatric observation medicine. Emerg Med Clin North Am 2001;19(1):239-254.

2. American College of Emergency Physicians Practice Management Committee. Management of observation units. Ann Emerg Med 1988;17(12):1348-1352.

3. Zun L. Observation Units: Boom or bust for Emergency Medicine. J Emerg Med 1990;8:15.

4. "Management of Observation Units: From the short term observation section of the American College of Emergency Physicians," available at www.acep.org.

5. Sinclair D, Green R. Emergency department observation unit: can it be funded through reduced inpatient admission? Ann Emerg Med 1998;32(6):670-675.

6. Mace SE. A national survey of observation units in the United States. Am J Emerg Med 2003;21:529-533.

7. LeDuc K, Haley-Andrews S, Rannie M. An observation unit in a pediatric emergency department: one children's hospital's experience. J Emerg Nursing 2002;28:407-413.

8. Martineau O, Martinot A, Hue V, et al. Effectiveness of a short-stay observation unit in a pediatric emergency department. Arch Pediatr 2003;10:410-406. 
9. Scribano PV, Wiley II JF, Platt K. Use of an observation unit by a pediatric emergency department for common pediatric illnesses. Pediatr Emerg Care 2001;17:321-323.

10. Zebrack M, Kadish H, Nelson D. The pediatric hybrid observation unit: an analysis of 6477 consecutive patient encounters. Pediatrics 2005;115(5):e535-e542.

11. Alpern ER, Calello DP, Windreich R, et al. Utilization and unexpected hospitalization rates of a pediatric emergency department 23-hour observation unit. Pediatr Emerg Care 2008; 24(9):589-594.

12. ACEP Practice Management Committee, American College of Emergency Physicians. Management of Observation Units. Irving TX: American College of Emergency Physicians; 1994.
13. Watson WA, Litovitz TL, Rodgers GC, et al. 2004 Annual Report of the American Association of Poison Control Centers Toxic Exposure Surveillance System. Am J Emerg Med 2005; 23:589-666.

14. Lemke T, Wang R. Emergency department observation for toxicologic exposures. Emerg Med Clin North Am 2001;19: $155-167$.

15. Dribben W, Welch J, Dunn D, et al. The utilization of emergency department observation units for the poisoned patient [Abstract]. J Toxicol/Clin Toxicol 1999;37:586.

16. Gummin DD, Butler JR, Roberts RR, et al. Utilization of an emergency department observation unit for acute intoxications [Abstract]. J Toxicol/Clin Toxicol 1999;37:586-587. 